\title{
Framework for Collaborative Application in Mobile Ad-hoc Network
}

\author{
Mr. Hardik S. Mehta, Dr. Dhaval R. Kathiriya \\ ${ }^{I}$ Research Scholar, School of Computer Science, RK University, Rajkot \\ ${ }^{2}$ Director IT, Anand Agricultural University, Anand
}

\begin{abstract}
With increase usage of mobile devices, there is a need to connect them together in meaningful way. Specifically, there a demand of application that enables collaboration among these mobile devices and their users. The users have different need and different way to collaborate using collaborative application while carrying the mobile devices. If developing time for a new application is too long then, it will most probably out of date by the time is will finished. This paper opens up with research motivations and questions, analyses the existing state of research in the field with their problem, and describes the approach in designing the proposed framework.
\end{abstract}

Index Terms: - Collaborative Application; Framework Designing; Functional Requirements; Mobile Ad-hoc Network;

\section{INTRODUCTION}

The mobile devices are one of the most popular consumer devices because of expanded in functionality from merely a device to dial numbers to personal digital assistants, and also they are cheaper in comparison to laptop computers so that we can carry and use them anywhere. Mobile applications these days are more flexible and can integrate the existing services by employing web-based interface. From last few years, the areas of Computer Supported Cooperative Work (CSCW) [Baecker93] and mobile computing [Duchamp94] was interested field from the personal use and commercial community. CSCW is defined as the study of how people work together or collaborate using technology of computer networking, and associated hardware, software, services and techniques [1]. The recent technical development has made it possible to work at a distance. Wide dissemination of mobile phones, increased capacity of computer networks, integration between telephony and computer systems are factors that work together to facilitate mobile computer supported co-operative work (Mobile CSCW).

Collaborative Application is based on the idea that people can share information. It is becoming more and more important everyday within all environments ranging from social to topical. The collaborative application can be used at emergency search and rescue operations, sharing of ideas and information in meeting or conferences, field survey operations at remote places, Group activity where no visual contact, security purpose, military operations and more. So people required different mobile applications for different purpose. The mobile application market is moving fast as more powerful phones are introduced. This short renewal time speeds up the adoption of new technology and creates a demand for applications utilizing new technology. If developing time for a new application is too long then, it will most probably out of date by the time is will finished.

This paper focus on need and designing a collaborative application framework using which different application can be developed rather than design a single application every time. It might require a significant larger amount of time to develop a framework rather than a single application. But a completed framework enables higher productivity and shorter development time for the actual applications, because of its capability of code and design reuse. This paper also discussing the related work done in this area and propose a prototyping model for developing collaborative application framework.

\section{RELATED WORK}

There are several projects that have developed frameworks or application related to collaboration. We will in this section present the most prominent projects.

The Anhinga project [4] is being developed by the Department of Computer Science, Rochester Institute of Technology. It aims to create a distributed computing infrastructure to support collaborative applications on wireless ad-hoc networks. It focuses on a decentralized structure and execution on mobile devices. Anhinga is based on Many-2-Many Invocation which eliminates the need for central servers and advanced routing, which makes it ideal for ad-hoc networks with low computational power. Even though the 
project is aimed at mobile devices, it uses J2SE. The main focus is towards PDAs like the Sharp Zaurus or the iPAQ which can run a complete $\mathrm{J} 2 \mathrm{SE}$ version.

The Jadabs [5] project is being developed by the Information and Communication Systems Research Group at the Swiss Federal Institute of Technology, Zurich. Its aim is to build a dynamic framework to enable mobile devices to insert, replace and remove applications at runtime. Communication is handled by an ad-hoc network and supports both UDP and Bluetooth connections. It doesn't support the collaborative application because its main focus on deploying the application runtime. It also uses the propagation method which require the use of CDC configuration.

JXTA (Maibaum \& Mundt, 2002) is an open-source framework for developing P2P applications. JXTA provides a set of protocols and APIs for general-purpose, computer-to-computer communication and is platform and network independent. JXME (Kawulok et al., 2005) is JXTA for Java 2 Micro Edition (J2ME) and is a lightweight implementation of JXTA for mobile devices. It is specifically aimed at devices without sufficient computation and/or communication resources to participate in the network on their own. The JXME implementation provides full JXTA functionality through the use of a relay host. There is also a JXME proxy less initiative, but there is currently no stable implementation. As JXTA does not have a pure peer-to-peer version working for J2ME.

Mobile Chedar (Auvinen et al., 2006) is a middleware being an extension to the Chedar peer-topeer network allowing mobile devices to access the Chedar network and communicate to other Mobile Chedars. The Mobile Chedar is implemented in J2ME and Bluetooth are used for communication. However, this approach suffers from having a single point of failure like client-server solutions because it uses gateway node which run on PC which provide gateway services to all the nodes.

Proem (Kortuem, 2002) is a framework for developing and deploying P2P collaborative applications in a mobile ad-hoc networking environment. The main objective of Proem is to provide a common framework for rapid development of applications for ad-hoc network environments. The framework is implemented in Java, and can be run on various wireless mobile devices. Proem is designed to be independent of underlying network transport protocols, and can be implemented on top of TCP/IP, HTTP, Bluetooth and others. The original Proem was based on a Java Standard Edition, limiting the devices to run Proem to powerful PDAs. There have been attempts to create a J2ME version of Proem that have not succeeded.

The JMobiPeer (Bisignano et al., 2005) framework provides support for discovery, group management and peer management. In addition JMobiPeer offers interoperability with JXTA. The implementation of JMobiPeer is based on J2ME. However, the actual execution of JMobiPeer has only been tested on emulators on standard PCs. This is probably because the framework has high requirements on CPU and memory. In addition, the framework does not reveal any details on the API or if they provide a pure or hybrid peer-to-peer solution.

Middleware LaCOLLA [Marques and Navarro 2006] has a fully decentralized peer-to-peer architecture and provides general purpose functionalities for building collaborative applications. LaCOLLA works well in networks with important signal stability, such as wired or fixed wireless (one-hop) networks. Framework design requires similar functions but for ad-hoc wireless (multi-hop) networks. Furthermore, LaCOLLA is not able to run on PDAs.

On the other hand, Nokia has developed a services-oriented framework that could be used to support mobile collaboration. This framework includes a set of APIs and an SDK (Software Development Kit) allowing developers to create service-oriented applications that act as consumers of Web services on mobile devices [Hirsch et al. (2006)]. Since mobile applications can just consume services, their autonomy is small because they require a service provider. It is unsuitable for our ad-hoc mobile work scenarios.

\section{PROBLEM WITH EXISTING APPROACHES}

This section will describe the limitation of all available existing framework which state that they are not suitable to develop the framework for collaborative application. (1) They can be used with complete J2SE platform [The Anhinga project] (2) Many of them is target the CDC platform and thus are not available on mobile phones [Jadabs, JXTA]. (3) Many of them is target for specific mobile phone [The Anhinga project PDA Sharp Zoros, iPAQ] (4) Many of them are very complex and requires higher processing and advanced P2P functionality [PeerWare, JXTA] (5) They focuses only on automatically information exchange between devices. [iCloud Project] (6) No stable implementation [JXTA - Maibaum \& Mundt, 2002] (7) They are more focused on Mobile Software than Collaborative Application [Medvidovic and Edwards - 2010]

\section{PROPOSED FRAMEWORK - MANCAF}

The proposed framework MANCAF is a framework for collaborative application in mobile ad-hoc network. The proposed framework is very useful to developer to design a collection of application for an entire domain. This framework will fulfill the diversify requirement of collaborative application of different users. The complete framework development is complex process and requires a significant amount of time but it provides 
higher productivity and shorter development time for the actual application, because of its capability of code and design reuse.



While designing a framework for different kind of application, we need to find out the requirements of application. The first step towards the development of framework will be the study of technologies like peer-topeer networks, mobile ad-hoc network and CSCW. Then the domain knowledge extracted from different research project which are currently available and different usage scenario are created to acquire the actual requirements. Then we build prototype of a framework based on the domain knowledge and the requirements. The framework is the implemented and tested over different technologies. At the last strength and weakness of the framework described based on the assumption made.

\section{REFERENCES}

[1] Brinck, Tom's CSCW and Groupware Page, http://www.infres.enst.fr/ vercken/multicast/cscw.html

[2] [Baecker93] Baecker, R., "Readings in Groupware and Computer Supported Cooperative Work", Morgan Kaufmann, San Mateo CA, ISBN 1-55860-241-0, 1993

[3] [Duchamp92] Duchamp, D., "Issues in Wireless Mobile Computing", Proc. Third Workshop on Workstation Operating Systems, Key Biscayne, Florida, U.S.A., pp 2-10, 1992

[4] http://www.cs.rit.edu/ anhinga/

[5] http://www.iks.inf.ethz.ch/projects/Jadabs

[6] Maibaum, N. and Mundt, T. (2002), JXTA: A Technology Facilitating Mobile Peer-To-Peer Networks, In International Mobility and Wireless Access Workshop (MobiWac'02), pages 7-13, Fort Worth, Texas, USA, 12 October.

[7] Kawulok, L., Zielinski, K., and Jaeschke, M. (2005), Trusted group membership service for JXME (JXTA4J2ME), Wireless And Mobile Computing, Networking And Communications, 2005. (WiMob'2005), pp. 116-121 Vol. 4, 22-24 Aug.

[8] Auvinen, A., Vapa, M., Weber, M., Kotilainen, N., and Vuori, J. (2006), Chedar: peer-to-peer middleware, Parallel and Distributed Processing Symposium, pp. 7, 25-29 April.

[9] Kortuem, G. (2002), Proem: a middleware platform for mobile peer-to-peer computing, ACM SIGMOBILE Mobile Computing and Communications Review, 6(4), pages 62-64.

[10] Bisignano, M., Di Modica, G., and Tomarchio, O. (2005), JMobiPeer: A Middleware for Mobile Peer-toPeer Computing in MANETs, In Proceedings of the First international Workshop on Mobility in Peer-ToPeer Systems (MPPS), June 6 - 10. 